\title{
Barred Galaxies: Intrinsic or Extrinsic?
}

\author{
M. Noguchi
}

Astronomical Institute, Tohoku University, Aoba, Sendai 980-77, Japan

\begin{abstract}
A unified picture is presented for the formation of bars in galaxies of different morphological types based on a simple model of the growth of galactic disks by gas infall from haloes. The infall timescale is found to be a key parameter which determines the dynamical property of the resulting disk. It is suggested that the bars in early-type disk galaxies have been formed in tidal interactions with other galaxies whereas those in late-type galaxies formed spontaneously due to gravitational instability (bar instability) in their disks.
\end{abstract}

\section{Introduction}

Galactic bars play a very important role in the evolution of disk galaxies, because they are quite common structures and have strong dynamical effects on the matter in the galactic disks due to their non-axisymmetric gravitational potentials. The phenomena associated with the redistribution of mass and angular momentum caused by a bar include gas infall which fuels nuclear starbursts and activity, and flattening of chemical abundance gradients across the disk.

Despite this importance, several fundamental questions remain still unanswered: when and how did actual bars form and how did they evolve with time? Especially important is to clarify which is the more dominant process of bar formation of the two possible mechanisms: 1) spontaneous formation due to global gravitational instability in the disk (e.g., Ostriker \& Peebles 1973) and 2) external triggering in tidal galaxy encounters (Noguchi 1987). In order to answer such questions with confidence, any theory of bar formation (especially of spontaneous bar formation) should be combined with a theory of galactic disk formation and evolution. Most previous studies on the process of spontaneous bar formation suffer from a serious defect that the numerical models employed in them lack evolutionary continuity to the disk formation process. The conventional initial condition widely adopted for bar instability simulations is a completely grown massive disk made solely of stars, which is highly artificial. Several lines of argument suggest gradual disk formation with a timescale of the order of several Gyr, which is longer than the typical growth time of unstable bars. Moreover, a significant portion of the disk mass is likely to remain gaseous for a long period, thus invalidating adoption of a purely stellar disk as initial conditions. Possible importance of the interstellar gas of even a small mass fraction has been dramatically demonstrated by the recent theoretical study by Shlosman \& Noguchi (1993) which shows that the highly dissipative gas effec- 
tively stabilizes the stellar disk against bar formation by creating discrete gas clumps of large individual masses, which scatter disk stars.

The purpose of the present study is to investigate the formation process of bars in the context of formation and evolution of galactic disks, taking into account this active aspect of interstellar gas discovered by Shlosman \& Noguchi (1993).

\section{Models of Disk Growth and Heating}

We follow here the conventional picture of disk galaxy formation in which galactic disks form gradually by gas infall in already virialized haloes. We consider a spherical halo with a radius $R(=10 \mathrm{kpc})$. The primordial gas initially included in this halo region gradually accretes to the disk plane and dissipatively builds up a galactic disk. The disk consists of the gas and the stars which formed from the gas contained in the disk. We assume that the stars and gas in the disk occupy a flat cylindrical region with a radius $R$ (the same as the halo) and a vertical height of $h_{s}(=600 \mathrm{pc})$ and $h_{g}(=200 \mathrm{pc})$, respectively. The region occupied by this system should be considered to represent the visible part of a disk galaxy within its optical radius. The physical state of each component (i.e., the halo, the stellar disk, and the gas disk) at a given time is specified by several global quantities whose values are averaged over the entire region of that component (i.e., we do not consider any structures in that component). The total mass of all three components is assumed to be conserved and denoted by $m_{t}\left(=10^{11} M_{\odot}\right)$.

With this simplification, the time evolution of masses in the gas and stellar disk components ( $m_{g}$ and $m_{s}$, respectively) is formulated as follows.

$$
\frac{d m_{g}}{d t}=-\frac{m_{g}}{\alpha}+\frac{m_{d}}{\beta} \exp \left(-\frac{t}{\beta}\right)-\frac{m_{g}}{\tau_{f r i}},
$$

and

$$
\frac{d m_{s}}{d t}=\frac{m_{g}}{\alpha},
$$

where $t$ is the time reckoned from the beginning of gas infall, which is here assumed to be $12 \mathrm{Gyr}$ ago. The first terms in the right hand side of both equations represent the effect of star formation and resulting gas consumption, where the star formation timescale, $\alpha$, is set to be proportional to the free-fall time of the gas component. Namely, $\alpha^{-1}=k\left(3 \rho_{g}\right)^{1 / 2}$, and the gas density $\rho_{g}=\frac{m_{g}}{\pi R^{2} h_{g}}$. The coefficient $k$ was set to be 0.04 so that the calculated models give star formation rates which fall in the observationally inferred range. The second term in equation (1) represents growth of the disk by gas infall from the halo region, the timescale of which is denoted by $\beta$. The final mass of the disk (i.e., the total mass of the matter which eventually accretes to the disk) is specified by $m_{d}$, which is set to be $50 \%$ of the total mass (i.e., $m_{d}=0.5 m_{t}$ ) in accordance with observational data. The third term in equation (1) is included here to make allowance for the depletion of disk gas due to gas flow to the galactic center caused by the dynamical friction acting on massive gas clumps, which is a back reaction of disk heating, as described below. 
The gas which has reached the disk plane is likely to cool and become gravitationally unstable when the $Q$-parameter (Toomre 1964) for the gas disk decreases to unity. This instability is a local one and should be discriminated from the bar instability which results from coherent response of the entire disk. When $Q=1$, the gas disk has only one neutrally stable wavelength $\lambda_{c}=\frac{2 \pi^{2} \Sigma_{g}}{\kappa^{2}}$ (for $G=1$ ), where $\kappa$ is the epicyclic frequency and the surface density of the gas is given by $\Sigma_{g}=\frac{m_{g}}{\pi R^{2}}$. Therefore, the typical mass of the gas clumps forming due to this instability is estimated to be $M_{c l}=\pi\left(0.5 \lambda_{c}\right)^{2} \Sigma_{g}=\frac{\pi^{5} \Sigma_{g}^{3}}{\kappa^{4}}$. In general, $M_{c l}$ is many orders of magnitude larger than individual stellar masses so that these clumps, while orbiting in the disk, effectively deflect orbits of disk stars. In other words, the gas clumps heat up the stellar disk dynamically. The degree of heating can be quantified by the increase in the velocity dispersion, $\sigma_{s}$, of the stellar random motions in the disk, which is given by

$$
\frac{d \sigma_{s}^{2}}{d t}=\gamma \sigma_{s}^{-2} .
$$

Here the coefficient $\gamma=\delta N_{c l} M_{c l}^{2}$ (Lacey 1984), where $N_{c l}$ is the surface number density of gas clumps and $\delta$ is determined by other characteristics. It is assumed here that $N_{c l} M_{c l} \sim \Sigma_{g}$, i.e., most of the gas participates the clumping.

Now equations (1), (2), and (3) complete a set of differential equations which determine the temporal evolution of a galactic disk. By integrating these equations numerically starting from the initial condition $\left(m_{g}=m_{s}=\sigma_{s}=0\right)$, we can get, as a function of time, the disk mass fraction $f_{d} \equiv\left(m_{g}+m_{s}\right) / m_{t}$, the gas mass fraction $f_{g} \equiv m_{g} / m_{t}$, and the velocity dispersion $\sigma \equiv\left(\sigma_{s}\right.$ divided by the characteristic rotational velocity). In the following, a set of $\left(f_{d}(t), f_{g}(t), \sigma(t)\right)$ is used to judge whether the stellar disk is unstable to spontaneous bar formation at a given time.

\section{Results}

We have run a series of models in which $\beta$ is varied from 10 to 80 in units of the dynamical time, which corresponds to $(0.5-4)$ Gyr if scaled to the present galactic parameters. This $\beta$ is only one important parameter in the present model and could be related to the Hubble morphological type as follows. The case with $\beta=0.5$ Gyr represents the limit of fast collapse in the sense that the disk growth timescale is comparable to the rotation period of the disk. This will correspond to the case of early-type galaxies which are considered to have formed in regions of a high gas density and hence have had a short free-fall time initially. On the other hand, the slowly growing model with $\beta=4 \mathrm{Gyr}$ will correspond to spirals of the latest type which are preferentially located in sparse regions in the universe.

Figure 1 shows time variation of several quantities of interest for different values of $\beta$. The model with a faster infall exhibits a higher maximum SFR at an earlier epoch but a lower SFR at present. This dependence on $\beta$ roughly corresponds to the observationally inferred change in the star formation history along the Hubble sequence. As $\beta$ decreases, a larger maximum value of $M_{c l}$ is attained at an earlier epoch, but the decline after the maximum is steeper. The 

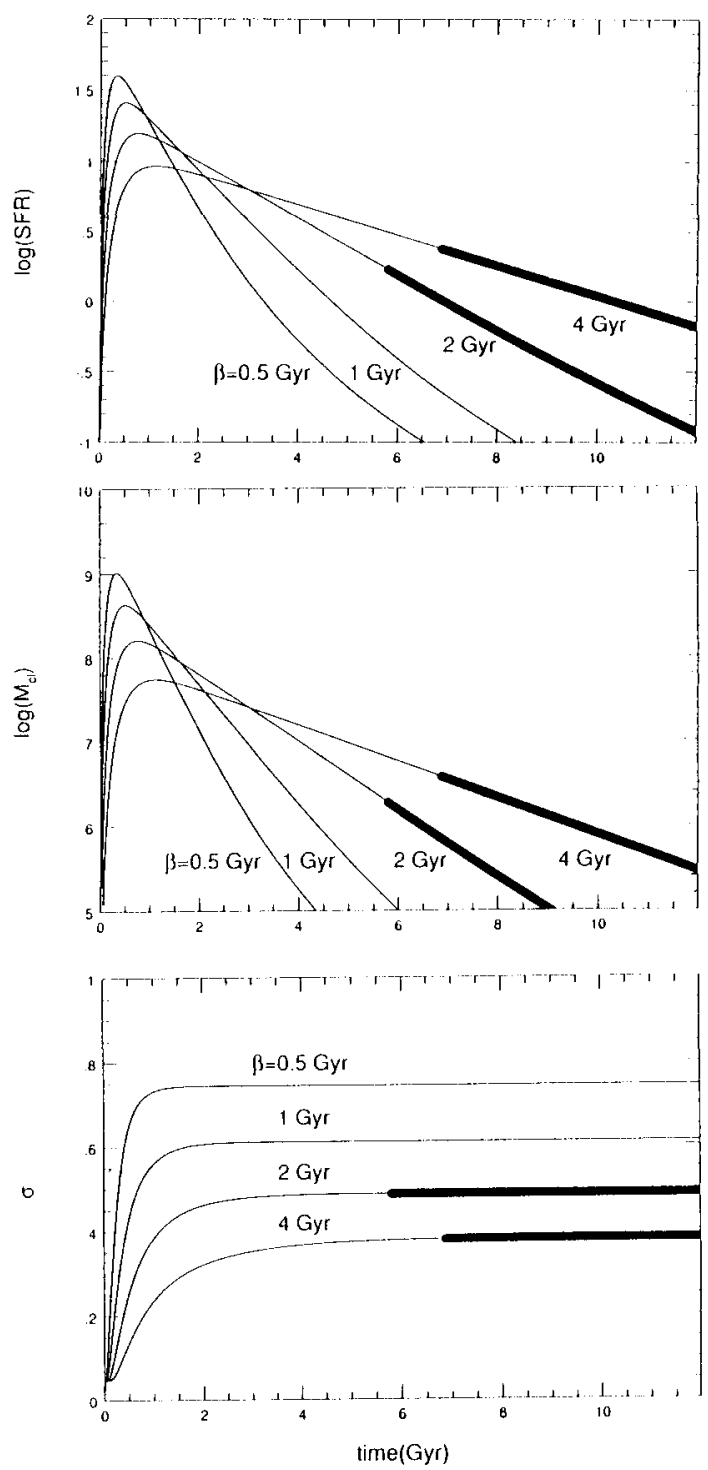

Figure 1. Time evolution of the model disks with different infall timescales $\beta$. From top to bottom, SFR (the star formation rate in units of $M_{\odot} \mathrm{yr}^{-1}$ ), the typical mass of the gas clumps formed in the disk (in units of $M_{\odot}$ ), and the velocity dispersion in the stellar disk (in units of the rotational velocity) are plotted. The phase when the stellar disk is bar-unstable is indicated by heavy lines. 


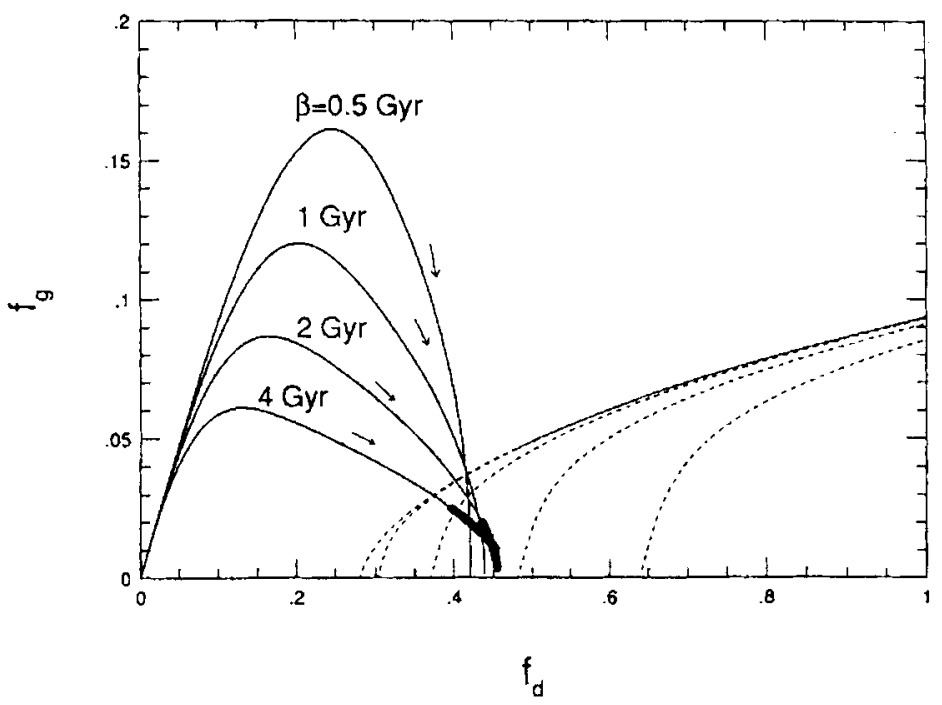

Figure 2. Trajectories on the $\left(f_{d}, f_{g}\right)$ plane of the model disks with different infall timescales $\beta$.

fastest growing model disk with $\beta=0.5 \mathrm{Gyr}$ develops very massive clumps with $M_{c l} \sim 10^{9} M_{\odot}$ in its most gas-rich phase. The more massive the gas clumps are, the more efficient the disk heating is. In the model with $\beta=0.5 \mathrm{Gyr}$, the stellar velocity dispersion becomes comparable to the rotational velocity.

The trajectory of each model is drawn in Figure 2. The criterion deduced by Shlosman \& Noguchi (1993) (their eq(24)) for the bar stability of the stellar disk in a two-component (stars plus gas) disk model can be represented as a surface defined on the $\left(f_{d}, f_{g}\right)$ plane which gives the critical value of the stellar velocity dispersion, $\sigma_{c r i t}$, required for stability as a function of $f_{d}$ and $f_{g}$. The contours of a constant $\sigma_{c r i t}$ are drawn in Figure 2 by dashed lines for $\sigma_{c r i t}$ (in units of rotational velocity) $=0,0.2,0.4,0.6$, and 0.8 (from left to right). The stellar disk of a given model is stable (unstable) if its actual $\sigma$ is larger (smaller) than $\sigma_{c r i t}$ for that model's $f_{d}$ and $f_{g}$. By tracing the time evolution of $f_{d}, f_{g}$, and $\sigma$, we can judge whether the stellar disk at any given epoch is bar-unstable or not.

In Figure 2, bar-unstable states are indicated by thick portions of the evolutionary tracks. It is seen that if $\beta$ is less than about $1 \mathrm{Gyr}$, the stellar disk remains stable during its whole evolution. When the disk growth is completed and most of its mass is converted into stars, the stellar disk component has already acquired sufficiently large random motions to assure stability due to the efficient heating in the gas-rich (i.e., $f_{g}>0.1$ ) phase. On the other hand, disk heating is not enough to damp bar instability in a slowly growing disk with $\beta>2$ Gyr because of the low maximum gas fraction attained. Its stellar component 
becomes bar-unstable at the instant when the total disk mass, $f_{d}$, exceeds the critical value determined by the gas mass fraction $f_{g}$ and the stellar velocity dispersion $\sigma$.

\section{Origins of Bars}

One natural and direct inference from the above results is that the late-type barred galaxies have resulted from bar instability in their disks whereas the bars in early-type galaxies have been formed in tidal interactions.

This scenario can find several independent supports as follows. One of these comes from environmental considerations. Observations suggest a morphological segregation that late-type disk galaxies are more frequently located in regions with low galaxy number densities, whereas early-type disk galaxies tend to reside in more crowded regions (e.g., Gisler 1980). Therefore late-type disk galaxies are considered to have had a smaller probability of encountering or colliding with other galaxies than early-type ones in their life. Then it is quite natural to think that the bars in late-type galaxies started to form spontaneously at the points when the disks became unstable without the help of external disturbances. On the other hand, early-type disk galaxies, which cannot form bars by themselves, had enough chance of tidal interactions, which excited bar structures in their disks.

The second and most strong support comes from numerical simulations which reveal a marked difference in the structures of tidal and spontaneous bars. Figure 3 shows a typical example of spontaneous bars. Here a stellar disk having the same mass as the spherical halo and relatively small random motions corresponding to $Q=1.5$ has been evolved without any external perturbations. The snapshots in Figure 3 indicate that the bar is quickly formed in three disk rotations and reaches a nearly steady state. It is noted that the surface density along the bar major axis decreases very rapidly with the radius. The density profile can be divided into two parts with transition occurring nearly at the bar ends. The profile in the inner part is steeper than that of the initial exponential disk shown by dashed lines, whereas the outer part shows a gentler decline, giving 'flaring' of the profile. A tidally-created bar (Figure 4) shows a remarkably different structure. In this case, the initial velocity dispersion in the disk has been set to $Q=2$, so that the disk did not develop a bar in isolated state. Otherwise this model has essentially the same characteristics as the model shown in Figure 3. This model galaxy was then made to have a close encounter with a point-mass perturber of the same mass on a parabolic orbit with a pericenter distance twice the disk radius. Figure 4 shows that a strong bar develops after the pericenter passage $(t=0)$ and lasts for a long time with little changes in strength and size. It is seen that the tidal bar has a much flatter density profile along the bar major axis than spontaneous bars. Moreover, sudden steepening in density gradient is seen near the ends of the bar, which gives 'shoulders' to the profile. Interestingly enough, this dichotomy in bar structure found in numerical models matches well the observational result for actual barred galaxies. Elmegreen \& Elmegreen (1985), based on extensive photometry, found that the observed bars fall into two groups according to the density profile along the bar major axis: 'flat' bars and 'exponential' bars, which respectively resemble the 

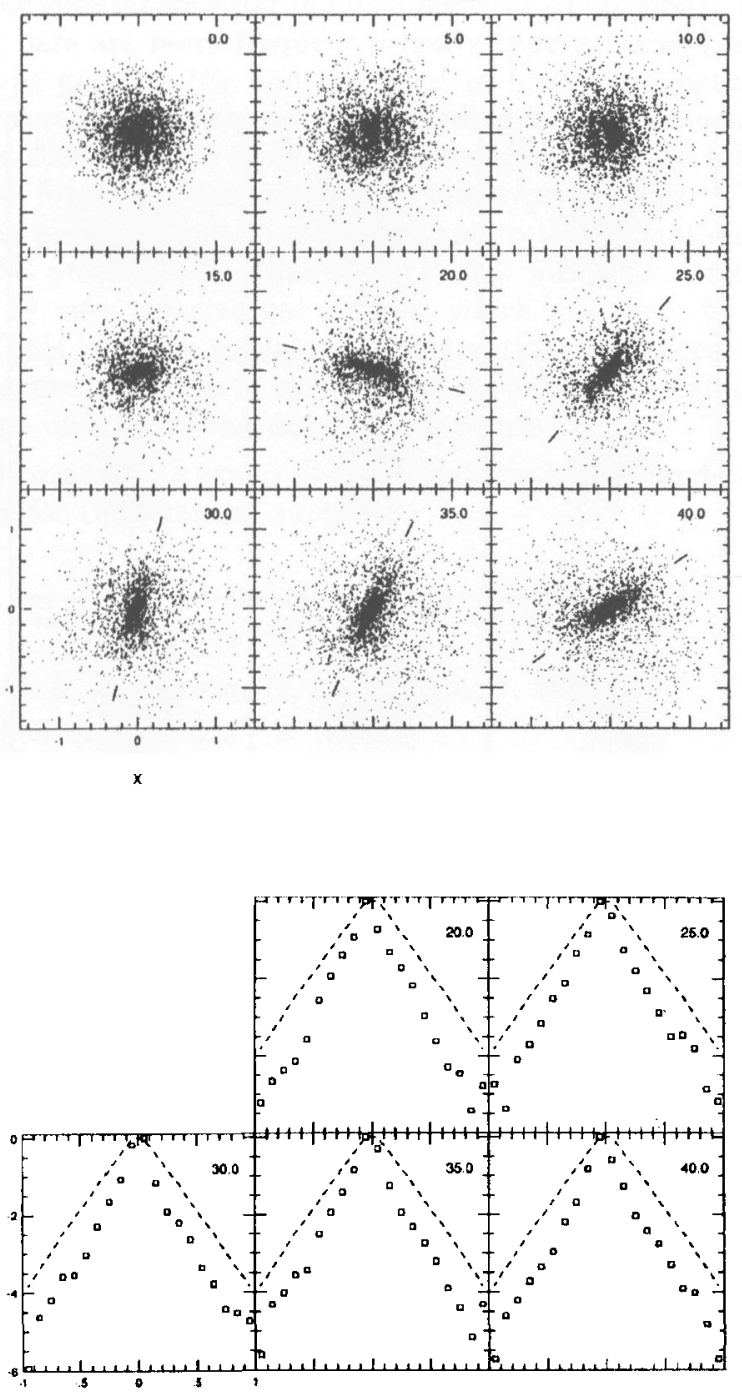

Figure 3. An example of spontaneous bars from numerical simulations. The upper panel gives snapshots of disk evolution in face-on view. Time $t$ (in units of $\sim 10^{8} \mathrm{yr}$ ) is indicated in the upper right corner of each frame. One rotation period of the outer disk edge is 6.28. The disk rotates counterclockwise. Squares in the lower panel give the surface density profile along the bar major axis, whose position angle is indicated by a pair of tick marks in the corresponding snapshot. The surface density is given in units of (negative) magnitude relative to the peak density. The dashed lines show the density profile of the initial axisymmetric disk. 

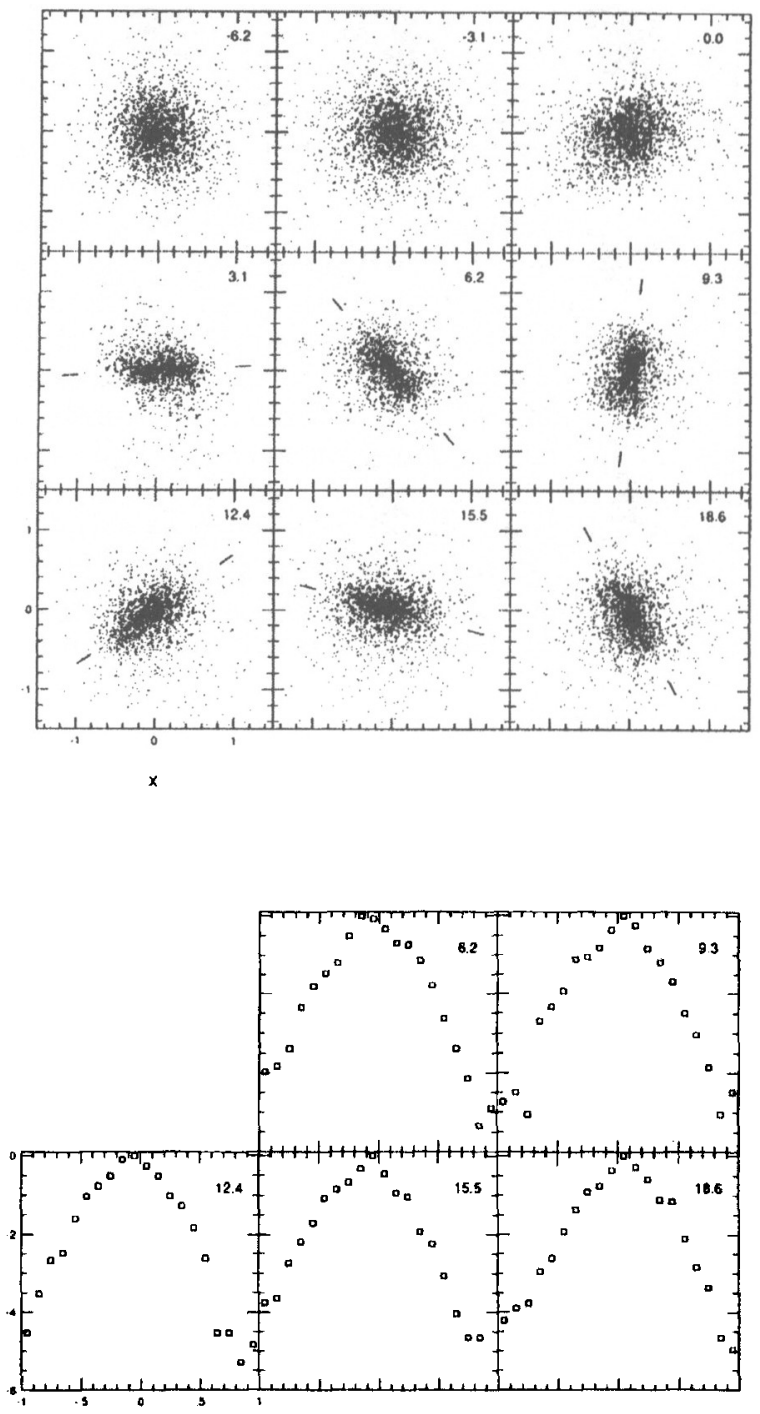

Figure 4. Same as Figure 3, but for an example of tidal bars. The perturber moves in the disk plane (i.e., the $\mathrm{x}$-y plane) and passes the pericenter $(x=2, y=0)$ at $t=0$ in the same direction as the disk rotation, i.e., counterclockwise. 
tidal bar and the spontaneous bar in our numerical simulations. Moreover, they found that flat bars are more frequent in early-type galaxies (i.e., $\mathrm{Sa}$ and $\mathrm{Sb}$ ), whereas late-type galaxies ( $\mathrm{Sc}$ and $\mathrm{Sd}$ ) tend to have exponential bars. Aided by the numerical result, this observational finding is quite naturally understood in our proposed scenario that early-type barred galaxies have tidal origin while spontaneous bar formation has occurred in late-type galaxies.

Finally, the present result may be relevant to the formation mechanism of thick disks. We propose here that a thick disk forms as a result of heating of a thin disk by very massive gas clumps, which are likely to form in a fast growing disk. This idea can explain the observational evidence that thick disk components are more common in early-type (i.e., bulge-dominated) disk galaxies than in late-type ones (e.g., van der Kruit \& Searle 1981).

Acknowledgments. I would like to thank Yoshida Foundation for Science and Technology for the financial support.

\section{References}

Elmegreen, B. G. \& Elmegreen, D. M. 1985, ApJ, 288, 438

Gisler, G. R. 1980, AJ, 85, 623

Lacey, C. G. 1984, MNRAS, 208, 687

Noguchi, M. 1987, MNRAS, 228, 635

Ostriker, J. P. \& Peebles, P. J. E. 1973, ApJ, 186, 467

Shlosman, I. \& Noguchi, M. 1993, ApJ, 414, 474

Toomre, A. 1964, ApJ, 139, 1217

van der Kruit, P. C., \& Searle, L. 1981, A\&A, 95, 116

\section{Discussion}

S. Ryder: Do you have plans to incorporate nucleosynthetic yields in your model, to track the evolution of abundance gradients?

M. Noguchi: No. It is not feasible in my simple model.

T. Hawarden: Have you considered how to account for the fact that many latetype galaxies (as many as those in early types) are not barred?

M. Noguchi: Such unbarred galaxies will be explained if the plausible distribution of the disk mass fraction $\left(m_{d}\right)$ to lower values is taken into account.

$F$. Combes: In your model, how can you explain that early-type galaxies have more frequently outer rings, that take a long time to form?

M. Noguchi: I did not consider outer rings. A gas dynamical simulation will be necessary to examine their formation.

J. Palouš: Do you consider in your model some type of gas recycling? Without it the gas would be consumed rapidly.

M. Noguchi: No. Probably due to this neglect, my models show unrealistically low star formation at the current epoch. However, this defect does not affect my 
conclusion because the dynamical property of the disk is primarily determined in early gas rich phases.

$H$. Ann: Is there any difference between the minor axis profile of the tidal and spontaneous bars?

M. Noguchi: I have not yet examined it. 